\title{
Ephedrine Infusion Instead of Crystalloid Preloading to Prevent Spinal Hypotension in Lower Abdominal Surgeries
}

\author{
Authors \\ Divya Madhu', ${ }^{1}$ Sheeja Krishnan ${ }^{2}, \operatorname{Rehana.B}^{3}$, P. Geethakumari ${ }^{4}$ \\ ${ }^{1,2,3}$ Assistant Professor. Dept of Anaesthesiology, Government Medical College, Thiruvananthapuram,
} Kerala

${ }^{4}$ Associate Professor, Dept of Anaesthesiology, Government Medical College, Thiruvananthapuram, Kerala Corresponding Author

Dr Sheeja Krishnan

Assistant Professor, Dept of Anaesthesiology, Government Medical College, Thiruvananthapuram, Kerala Telephone Number: 9447525228, Email: sheejakrishnan@yahoo.com

\begin{abstract}
Spinal anesthesia is one of the most popular techniques for lower limb and lower abdominal procedures. Various prophylactic methods like prehydration, head up position and vasopressors have been used to minimize hypotension associated with it. In our study, we used prophylactic Ephedrine infusion to prevent hypotension, in spinal anaesthesia. 80 ASA I and II patients aged 20 to 60 years were randomly allocated into two equal groups. Group 1 received prophylactic intravenous infusion of Ephedrine at the rate of $5 \mathrm{mg} / \mathrm{min}$ for the first two minutes followed by $1 \mathrm{mg} / \mathrm{min}$ for the next 18 minutes following spinal block. Group 2 received crystalloid preloading at $15 \mathrm{ml} / \mathrm{kg}$ over 15 to 20 minutes before spinal block. Patients in both the groups were given maintenance infusion of crystalloid at the rate of $5 \mathrm{ml} / \mathrm{kg}$ for 20 minutes after spinal block and according to requirement thereafter. Hypotension was taken as more than $20 \%$ fall in Systolic BP from baseline. The occurrence of any side effects was also monitored. Incidence of hypotension was significantly higher in the control group (82.2\%) vs Ephedrine group (22.5\%); $p<0.001$. Additional Ephedrine boluses needed to treat hypotension was significantly lower in Ephedrine group $(p<0.001)$. No side effects were noted in $67.5 \%$ patients in Ephedrine group compared to $62.5 \%$ for control group. There was $7.5 \%$ incidence of reactive hypertension( $>140 / 90 \mathrm{~mm} \mathrm{hg}$ ) and $20 \%$ had tachycardia (>100/ $\mathrm{min})$ in the Ephedrine group. Administration of prophylactic Ephedrine infusion is a suitable alternative to preloading with crystalloids. Incidence of hypotension is lower and BP maintains a more stable trend. But it is safe to avoid it in patients with preexisting tachycardia or hypertension.

Keywords: ephedrine, crystalloids, preloading, spinal anaesthesia, lower abdominal surgeries.
\end{abstract}

\section{Introduction}

On August $16^{\text {th }}, 1898$, a German Surgeon, Karl August Bier performed the first spinal anaesthesia $^{1}$ More than a century has passed and even today, it is one of the most popular techniques for lower limb and lower abdominal procedures. Its advantages revolve around its simplicity of administration, faster onset, profound sensory and motor blockade, adequate muscle relaxation, better control of airway with 
reduced risk of gastric aspiration \& airway obstruction and its minimal side effects ${ }^{2}$.

In spinal anaesthesia it is the blockade of anterior and posterior nerve roots which produces the typical effects. Local anaesthetic solution acts on autonomic, sensory and motor fibres. The autonomic being the most sensitive and the motor fibers the least sensitive. Three sites of action of local anaesthetic placed in the subarachnoid space are identified in the order of importance (a) On the nerve roots of spinal cord (b) On dorsal root ganglia and posterior-anterior horn synopsis (c)limited and incomplete action in spinal cord parenchyma on ascending and descending tract ${ }^{3}$. The physiological effects of central neuraxial blocks results in decreased heart rate and blood pressure ${ }^{4}$.

The major physiological concern about spinal anesthesia center around the hypotension associated with the block. Hypotension occurs as a result of blockade of pre ganglionic sympathetic fibres, leading to a reduction in systemic vascular resistance, increase in venous capacitance and venous pooling which reduces the cardiac output ${ }^{5}$. The incidence of hypotension has been reported to be very high in an untreated group undergoing surgery with spinal anesthesia ${ }^{6}$ Despite advancements in the management, hypotension continues to be a significant problem during spinal anaesthesia. Various prophylactic measures such as prehydration, positioning and vasopressors have all been used to minimize hypotension following spinal anaesthesia ${ }^{7}$ Compensatory volume expansion is routinely practiced as a measure for prevention of spinal hypotension. However there is accumulating evidence to suggest that this technique, may not only be ineffective but also could be potentially dangerous to those with marginal cardiovascular reserve. Sustained infusion of large volume of crystalloids over a short period of time carries a risk of pulmonary edema in patients of poor myocardial function. It also aggravates post-operative urinary retention. Prophylactic preloading is questionable in normovolemic patients also as it is found to be not only less effective in preventing hypotension, it can also reduce the arterial oxygen content and thus decrease oxygen delivery to the tissues 89 Various colloid solutions including albumin have also been tried for pre-loading in spinal anaesthesia. The incidence of hypotension has been shown to be lower but not completely eliminated. These solutions are also very expensive and it may not be cost effective to use them as a routine in our population. One study even suggests the possibility of increased coagulability and increased incidence of DVT with crystalloid preloading. Reduction of blood loss (30-40\%) with spinal anaesthesia has been reported. ${ }^{10}$ This may be due to lack of sympathetic activity from pain, avoidance of reactive arterial hypertension and avoidance of increased airway and thoracic pressures ${ }^{11}$ Fluid preloading before spinal anaesthesia is also time consuming and may be inappropriate in the setting of emergency surgery. A patient who would have otherwise received a spinal anaesthesia may be administered a general anaesthesia to save time required for preloading. Thus some studies had shown that routine crystalloid preloading before subarachnoid block is effective in decreasing the severity of hypotension ${ }^{12}$, and some studies showed that preloading has limited effect on the incidence of hypotension ${ }^{13}$

Considering the magnitude of the problem and the afore mentioned disadvantages of preloading, there is a quest for an alternative to preloading. In the recent years reports have suggested that prophylactic use of vasopressors is highly safe and efficient in eliminating hypotension following subarachnoid block. Ephedrine is a commonly used vasopressor to treat hypotension during spinal anaesthesia. It directly stimulates both alpha and beta receptors and also releases norepinephrine from sympathetic nerve endings to increase cardiac output, heart rate, systolic and diastolic pressures ${ }^{14}$ Ephedrine increases blood pressure both by increasing cardiac output and by peripheral vasoconstriction. Prophylactic ephedrine has been found to prevent hypotension during spinal anaesthesia in various studies. 
We conducted this study to evaluate and compare the relative efficacy of prophylactic ephedrine infusion versus crystalloid preloading for the prevention of hypotension during spinal anaesthesia in patients undergoing lower abdominal surgery.

\section{Materials and Methods}

We recruited 80 Patients of ASA Grade I and II undergoing elective lower abdominal surgery in the age group 20 - 60 years after obtaining written informed consent and approval from institutional research and ethical committees. Patients with spinal deformities, previous spine surgeries, coagulation abnormalities, serious pulmonary or cardiovascular diseases, patients with intraabdominal mass/ pregnancy, those with documented drug allergy to bupivacaine and ephedrine and those in whom anticipated operative blood loss is greater than $10 \%$ of blood volume were not recruited into the study.

A prior sample size calculation was done and the sample size was fixed at 40 per each limb of the study ${ }^{15}$. We randomly allocated the patients into two groups by computer generated random number sampling.

Group1 Patients did not receive fluid till the administration of spinal block; but received prophylactic ephedrine infusion after the spinal block.

Group 2 Patients were preloaded with $15 \mathrm{ml} / \mathrm{kg}$ of crystalloid infused rapidly over a period of $15-20$ minutes before institution of spinal block.

Routine blood and urine examinations were done for all patients. Age, sex, weight, height, type of surgery noted. Baseline variables, heart rate, diastolic and systolic blood pressures were recorded. Emergency medicines were kept ready prior to starting the procedure. All patients were pre medicated with Tab. Diazepam $0.2 \mathrm{mg} / \mathrm{kg}$ and Tab. Ranitidine $150 \mathrm{mg}$ previous night and early morning on the day of surgery. Stopping rule was any serious clinical adverse effect.

Spinal anaesthesia was carried out under strict aseptic technique, in right lateral position. After skin infiltration with lignocaine, using a $23 \mathrm{G}$ spinal needle, at L3 - L4 vertebral interspace, $0.5 \%$ Bupivacaine heavy was given intrathecally. The patients were immediately placed in supine position. The surgery was allowed to start when sub arachnoid block level of T6 as assessed by loss of pin prick sensation was achieved.

(a) In Ephedrine group of patients, after turning the patients supine, ephedrine infusion prepared in $0.9 \% \mathrm{NS}$ in a concentration of $1 \mathrm{mg} / \mathrm{ml}$ was started prophylactically at a rate of $5 \mathrm{mg} /$ minute for the first 2 minutes $(5 \mathrm{ml} / \mathrm{min}$ ) and then at a rate of $1 \mathrm{mg} /$ minute $(1 \mathrm{ml} / \mathrm{min})$ for the next 18 minutes.

(b) Reference therapy -All patients in the crystalloid pre-loading group were preloaded with $15 \mathrm{ml} / \mathrm{kg}$ of crystalloid infused rapidly over a period of 15 - 20 minutes before institution of spinal block.

All patients in both the groups were given infusion of crystalloid after spinal block at a rate of $5 \mathrm{ml} / \mathrm{kg}$ for the first 20 minutes and after that according to the need of the patients.

In this study, hypotension is taken as more than $20 \%$ fall in systolic blood pressure from baseline. Hypotension if any occurring after sub arachnoid block in both the groups were treated promptly with ephedrine $6 \mathrm{mg}$ intravenous bolus, repeated if necessary.

Pulse rate, systolic and diastolic blood pressures were recorded every 2 minutes for the first 10 minutes and then every 10 minutes till the end of surgery. Total dose of ephedrine given to patients was recorded.

In addition, all patients were monitored for any side effects of ephedrine.

Primary outcome measure was hypotension, defined as $>20 \%$ fall in systolic BP from baseline value. Secondary outcome measure was the occurrence of any adverse effects.

Statistical analysis was done using R and strata 15 Software programs. Descriptive statistics is calculated for baseline variables. Mean, median and SD calculated for continuous variables such as age. Proportions calculated for categorical variables .Primary analysis is based on ' Intention To Treat Analysis'. (ITT). Since the primary 
outcome measures are categorical variables, Chi square test is used for the outcome analysis. Student $t$ test is used to assess the significance of difference in the mean values of a variable between the two groups. A p value $<0.05$ is considered significant. Observations are represented both numerically and graphically.

\section{Results}

In the present study, there were 80 patients 40 each in the ephedrine group and control group. The median age of the study population was 39(IQ 26-47). There were a male preponderance in the study group with $60 \%$ compared to the females. The mean weight was $58.4 \pm 7.3 \mathrm{~kg}$. Baseline demographic features were comparable across the two groups excepts for the heart rate(table1). Systolic, diastolic and mean arterial pressures were consistently higher in the ephedrine group compared to the control group at all time points we measured. In the case of heart rate, there was a statistically significant increase in the ephedrine group till 90 minutes compared to the control group. Thereafter there was no statistically significant difference in the heart rate between both groups.

Table 1: Baseline comparison between the two groups

\begin{tabular}{lcccc} 
& [ALL] N=80 & Control N=40 & Ephedrine N=40 & p.overall \\
\hline Age & $39.0[26.0 ; 47.0]$ & $39.0[26.0 ; 46.0]$ & $39.5[27.5 ; 48.0]$ & 0.408 \\
Gender : & & & & 0.494 \\
$\quad$ female & $32(40.0 \%)$ & $18(45.0 \%)$ & $14(35.0 \%)$ & \\
$\quad$ male & $48(60.0 \%)$ & $22(55.0 \%)$ & $26(65.0 \%)$ & \\
Weight & $58.4(7.30)$ & $58.2(6.86)$ & $58.6(7.79)$ & 0.773 \\
Systolic BP & $124[118 ; 130]$ & $126[118 ; 130]$ & $122[115 ; 130]$ & 0.250 \\
diastolic BP & $80.0[76.0 ; 82.0]$ & $80.0[78.0 ; 82.0]$ & $80.0[74.0 ; 80.0]$ & 0.250 \\
Mean arterial pressure & $95.0[89.0 ; 97.0]$ & $95.5[91.0 ; 97.0]$ & $93.0[87.8 ; 98.0]$ & 0.106 \\
Heart rate & $75.0[72.0 ; 80.0]$ & $72.0[72.0 ; 74.0]$ & $80.0[78.0 ; 88.0]$ & $<0.001$
\end{tabular}

Incidence of hypotension, the main outcome measure was significantly higher in the control group (82.2\% vs $22.5 \%$ ) with a $\mathrm{p}$ value less than 0.001 (figure1 ).

Hypotension in the control and ephedrine group

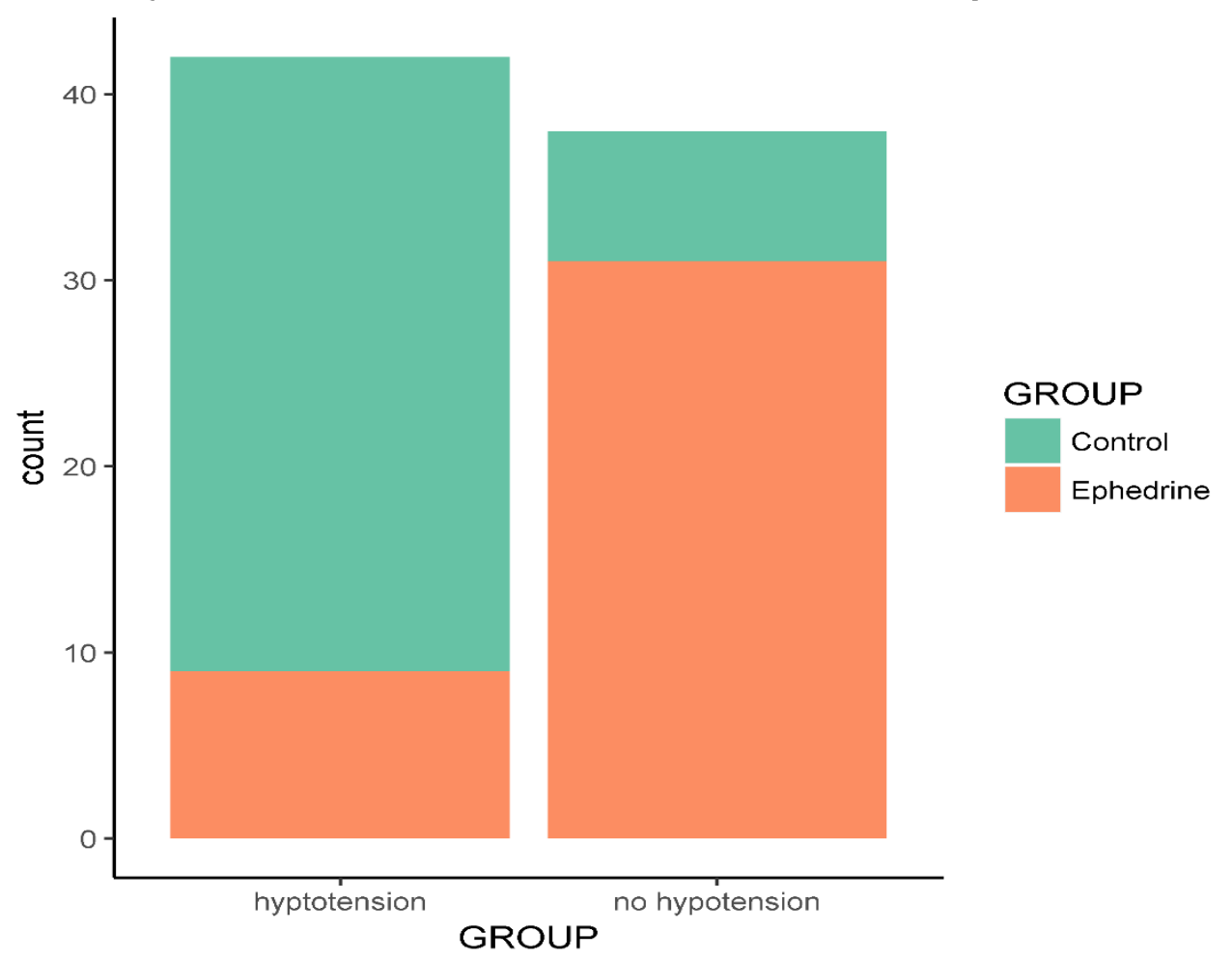


The number of additional ephedrine boluses administered to counteract the hypotension was significantly lower in the ephedrine group compared to the control group ( $\mathrm{p}$ value $<0.001$ )

(table 2)

Table 2: Outcome measures in the ephedrine and control group.

\begin{tabular}{lcccc} 
& [ALL] N=80 & Control N=40 & Ephedrine N=40 & p.overall \\
\hline Hypotension: & & & & $<0.001$ \\
Hypotension & $42(52.5 \%)$ & $33(82.5 \%)$ & $9(22.5 \%)$ & \\
No hypotension & $38(47.5 \%)$ & $7(17.5 \%)$ & $31(77.5 \%)$ & \\
Side effects: & & & & $<0.001$ \\
Hypertension & $3(3.75 \%)$ & $0(0.00 \%)$ & $3(7.50 \%)$ & \\
Nausea & $17(21.2 \%)$ & $15(37.5 \%)$ & $2(5.00 \%)$ & \\
No side effects & $52(65.0 \%)$ & $25(62.5 \%)$ & $27(67.5 \%)$ & \\
Tachycardia & $8(10.0 \%)$ & $0(0.00 \%)$ & $8(20.0 \%)$ &
\end{tabular}

Overall there were no side effects reported for incidence of tachycardia and hypertension were $67.5 \%$ of patients in the ephedrine group compared to $62.5 \%$ for the control group. The higher in the ephedrine group compared to the control group. $(\mathrm{p}<0.001)($ table2) (figure 2$)$

\section{Side effects in Ephedrine and Control group}

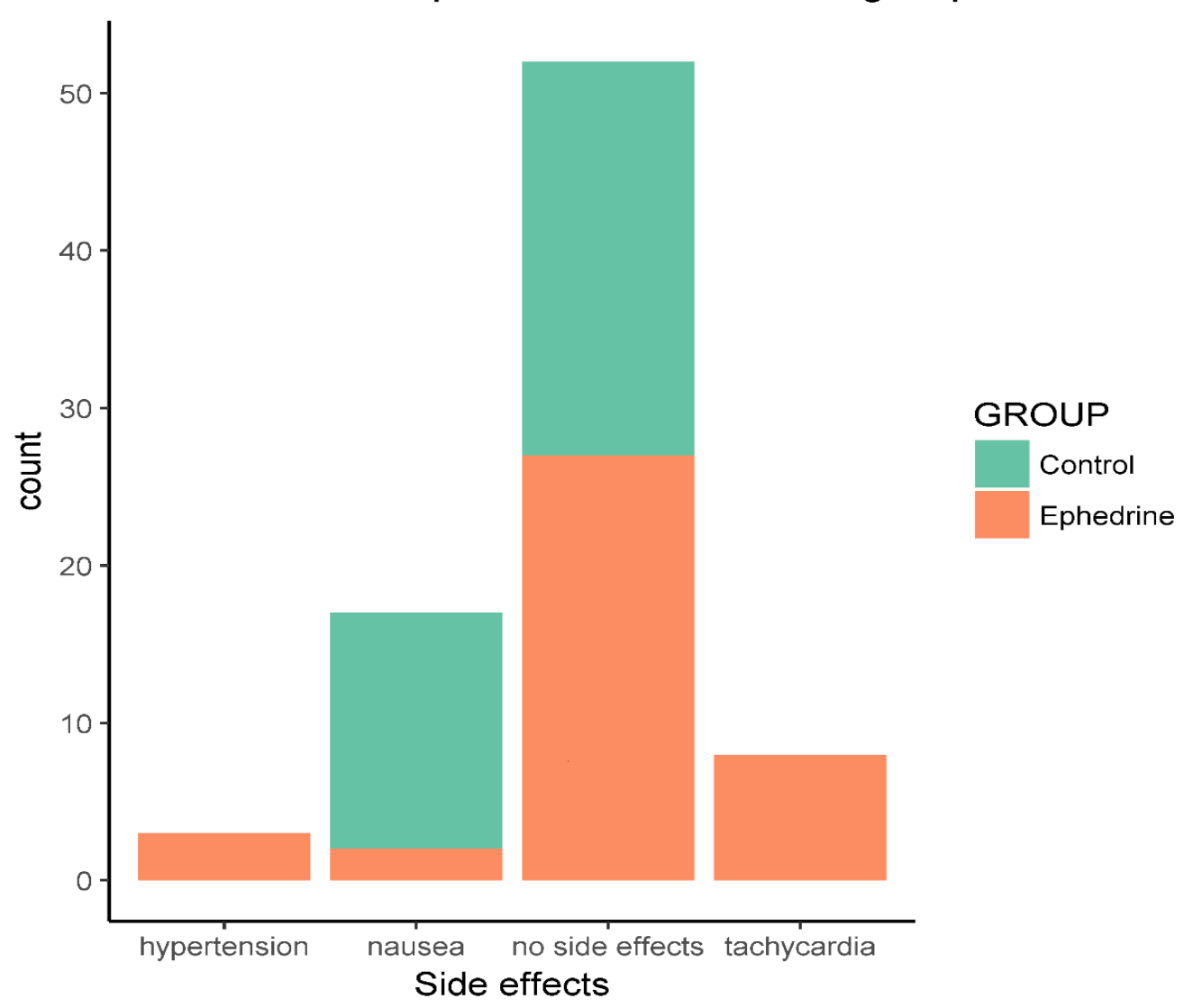

\section{Discussion}

The main objective of this study was to find out whether prophylactic ephedrine infusion reduces the occurrence of hypotension in patients undergoing spinal anesthesia and hence whether it can be a suitable alternative to crystalloid preloading. In this randomized controlled study we compared the haemodynamic variations of two groups of patients undergoing spinal anesthesia for elective lower abdominal and lower limb surgeries. Here, prophylactic Ephedrine infusion reduced the incidence of hypotension following subarachnoid block. The patients in the Ephedrine group had significantly lesser requirement of intravenous Ephedrine bolus intraoperatively as compared to patients in the control group and incidence of side effects like nausea and vomiting were minimal in the ephedrine group. Though 
total side effects were less in ephedrine group, a few people in ephedrine group had tachycardia and reactive hypertension.

Similar results were reported in other studies. The study by Ahmed et al concluded the same. However there was no tachycardia in the ephedrine group. In our study, there was a statistically significant increase in the heart rate in the ephedrine group compared to control group till 90 minutes. However the heart rate in the ephedrine group in the baseline itself was higher compared to control group. Apart from this, another reason could be due to release of nor epinephrine indirectly by the ephedrine administered. Idehen et all concluded no difference between the group after 10 minutes. However the patient population in their study was with different physiology ${ }^{16}$.

9 people in ephedrine group (22.5\%) had hypotension compared to 33 people $(82.5 \%)$ in crystalloid group, $\mathrm{p}$ value $<0.001$ and this is statistically highly significant.

Similar findings were reported by Kang et al who found $79 \%$ incidence of hypotension in the fluid preloading group, while the group receiving ephedrine had hypotension only in $9.1 \%$ of cases ${ }^{14}$.

In Bhattacharya et al study, they observed $60 \%$ and $20 \%$ incidence of hypotension in crystalloid preloading and ephedrine infusion group respectively ${ }^{17}$.

A study conducted by S.Kundra, V.Abraham and Afzal showed $80 \%$ incidence of hypotension in the preloading group compared to $26.67 \%$ hypotension in the ephedrine group ${ }^{15}$.

Gutscheetal found that administration of prophylactic ephedrine decreased the incidence of hypotension as well as that of nausea and vomiting.$^{18}$ Nakayama et al found that the hepatic blood flow is decreased due to spinal anaesthesia and can be attenuated by ephedrine administration ${ }^{19}$ Gajraj et al found that the incidence of hypotension was $55 \%$ in the crystalloid group and $22 \%$ in the infusion group ${ }^{20}$ They concluded that a prophylactic ephedrine infusion is effective for minimizing and managing hypotension associated with spinal anaesthesia and compares favorably with crystalloid administration in terms of efficacy and incidence of side effects. Ergolu $\mathrm{F}$ et al concluded in their study that a prophylactic oral dose of ephedrine 50 $\mathrm{mg}$ is effective for minimizing and managing spinal anaesthesia induced hypotension during transurethral prostatectomy ${ }^{21}$

In our study the fluid preloading group required significantly higher mean ephedrine boluses to treat hypotension $(11.1 \mathrm{mg})$ compared to the ephedrine group (8mg). The crystalloid preloading group received a mean total dose of $9.2 \mathrm{mg}$ and ephedrine group received a mean total dose of $29.8 \mathrm{mg}$. $(\mathrm{p}<0.001)$.

Gajraj et al (1993) in his study found that crystalloid group received a mean of $15.2 \mathrm{mg}$ ephedrine and ephedrine group received a mean of $37.4 \mathrm{mg}$. The crystalloid group also received more boluses of ephedrine than fluid infusion group ${ }^{18}$. Bhattacharya et al showed the mean dose of ephedrine in crystalloid group was $15.3 \pm 19.2 \mathrm{mg}$ and in ephedrine infusion group, total mean dose required was $37.6+10.2 \mathrm{mg}$. the mean number of ephedrine boluses were $1.2 \pm 1.6$ and $0.2 \pm 0.3$ respectively in crystalloid and ephedrine infusion group $^{17}$. In Kundra study mean total dose of ephedrine in crystalloid group was $10.3 \mathrm{mg}$, while in ephedrine group was $28.93 \mathrm{mg}^{15}$.

In ephedrine group, $67.5 \%$ had no side effects compared to $62.5 \%$ in crystalloid group. So, total side effects were less in ephedrine group.

None of the patients in crystalloid group developed reactive hypertension (> 140/90mm $\mathrm{Hg}$ ) in comparison to $7.5 \%$ incidence in ephedrine group. Also, $20 \%$ of ephedrine group had tachycardia (>100/min) whereas none of the patients in control group developed tachycardia. The Kundra study, 52 had $13.33 \%$ incidence of reactive hypertension in the ephedrine group compared to none in crystalloid group.

Incidence of nausea and vomiting were less in ephedrine infusion group. Nausea had $37.5 \%$ incidence in crystalloid group and $5 \%$ in ephedrine group. Major cause of nausea and vomiting is the hypotension, leading to reduction 
in medullary blood flow to the chemoreceptor trigger zone. Ephedrine is commonly employed to increase the mean arterial pressure which presumably improves the medullary blood flow, thus minimizing these symptoms. A high degree of vagal tone in the perioperative period may also add to the incidence of nausea and vomiting and Ephedrine may minimize these symptoms by increasing the sympathetic tone. Kang et al in his study also found lesser frequency of nausea and vomiting in the ephedrine group ${ }^{14}$.

Bhagat et al studied effect of preloading alone, ephedrine iv alone and combination of preloading and iv ephedrine for prevention of hypotension and concluded that combination of preloading and iv ephedrine was more effective than any measure alone and that preloading alone was the least effective.

None of the patients in the ephedrine group had any symptoms suggestive of cerebral stimulation like restlessness or anxiety ${ }^{5}$.

Most of the studies about the beneficial effects of ephedrine as a prophylactic measure instead of preloading with crystalloid were conducted in Cesarean section. Our study is one of the few studies which looked into the beneficial effects of ephedrine as an alternative to preloading in patients undergoing lower abdominal surgeries and under spinal anesthesia

Our study has a few limitations. In this randomized control study, the baseline heart rate in the ephedrine group was higher compared to the control group. This must have influenced the analysis and interpretation of increased prevalence of heart rate in the ephedrine group. As subgroup analysis was not planned at the time of sample size calculation in protocol stage, we have not attempted a sub group analysis to tackle the baseline difference in the heart rate. In addition, the lack of allocation concealment may have influenced our study. As a result, there was a potential chance for selection bias in this study. Future studies taking into account these repercussion in the study need to be planned.

\section{Conclusion}

Our study shows that administration of prophylactic ephedrine infusion for prevention of post spinal hypotension is a suitable alternative to preloading with crystalloids. It has been found to be a simple and effective method of reducing the incidence of hypotension after subarachnoid block. The incidence of hypotension was found to be lower and the blood pressure maintained a more stable trend when intravascular ephedrine infusion was used. It saves time in emergency surgery and would be economical in the Indian context. However, it is safer to avoid it in a patient with preexisting tachycardia or hypertension.

\section{Source of Support: Self}

\section{References}

1. Wulf HFW. The Centennial of Spinal Anesthesia. Anesthesiology 1998; 89(2): 500-6.

2. Hawkins JL, Arens JF, Bucklin BA, et al. Practice guidelines for obstetric anesthesia: An updated report by the American Society of Anesthesiologists Task Force on obstetric anesthesia. Anesthesiology 2007; 106(4): 843-63.

3. Collins VJ. Principles of anesthesiology: general and regional anesthesia. 3rd ed ed. Philadelphia: Lea \& Febiger; 1993.

4. Miller's anesthesia. 6th ed ed. New York: Elsevier/Churchill Livingstone; 2005.

5. Malhotra HBDK. Evaluation of preloading and vasoconstrictors as a combined prophylaxis for hypotension during subarachnoid anaesthesia. Indian Journal of Anaesthesia 2004; 48(4): 299-303.

6. Burns SM, Cowan CM, Wilkes RG. Prevention and management of hypotension during spinal anaesthesia for elective Caesarean section: a survey of practice. Anaesthesia 2001; 56(8): 777-98.

7. Lee A, Kee WDN, Gin T. Prophylactic ephedrine prevents hypotension during spinal anesthesia for Cesarean delivery but does not improve neonatal outcome: a 
quantitative systematic review. Canadian Journal of Anesthesia/Journal canadien d'anesthésie 2002; 49(6): 588-99.

8. Venn PJH, Simpson DA, Rubin AP, Edstrom HH. Effect Of Fluid Preloading On Cardiovascular Variables After Spinal Anaesthesia With Glucose-Free $0.75 \%$ Bupivacaine. BJA: British Journal of Anaesthesia 1989; 63(6): 682-7.

9. Coe AJ, Revanäs $\mathrm{B}$. Is crystalloid preloading useful in spinal anaesthesia in the elderly? Anaesthesia 1990; 45(3): 2413.

10. d'A Stanton-Hicks M. A Study Using Bupivacaine For Continuous Peridural Analgesia In Patients Undergoing Surgery Of The Hip. Acta Anaesthesiologica Scandinavica 1971; 15(2): 97-106.

11. Bromage PR. Epidural Air Bubbles and Frothy Syllogisms. Anesthesia \& Analgesia 1988; 67(1): 93.

12. Clark RB, Thompson DS, Thompson $\mathrm{CH}$. Prevention of Spinal Hypotension Associated with Cesarean Section. Anesthesiology 1976; 45(6): 670-3.

13. Jackson R, Reid JA, Thorburn J. Volume preloading is not essential to prevent spinal-induced hypotension at Caesarean section. British Journal of Anaesthesia 1995; 75(3): 262-5.

14. Kang YG, Abouleish E, Caritis S. Prophylactic Intravenous Ephedrine Infusion during Spinal Anesthesia for Cesarean Section. Anesthesia \& Analgesia 1982; 61(10): 839???42.

15. Kundra S, Abraham V, Afzal L. Prevention of hypotension during spinal anaesthesia for caesarean section: Ephedrine infusion versus crystalloid preloading. Journal of Anaesthesiology Clinical Pharmacology 2008; 24(4): 433-6.

16. Idehen H, Amadasun F, Ekwere I. Comparison of intravenous colloid and colloid-crystalloid combination in hypotension prophylaxis during spinal anesthesia for cesarean section. Nigerian Journal of Clinical Practice 2014; 17(3): 309-13.

17. Bhattacharya D, Chowdhury M, Biswas B, Gazi N, Rudra A, Banerjee A. Comparison of an ephedrine infusion with crystalloid administration for prevention of hypotension during spinal anaesthesia for elective caesarean section. Indian Journal of Anaesthesia 2001; 45(4): 290-93.

18. Gutsche BB. Prophylactic Ephedrine Preceding Spinal Analgesia for Cesarean Section. Anesthesiology 1976; 45(4): 4625.

19. Nakayama M, Kanaya N, Fujita S, Namiki A. Effects of Ephedrine on Indocyanine Green Clearance During Spinal Anesthesia. Anesthesia \& Analgesia 1993; 77(5): 947???9.

20. Gajraj NM, Victory RA, Pace NA, Van Elstraete AC, Wallace DH. Comparison of an Ephedrine Infusion with Crystalloid Administration for Prevention of Hypotension During Spinal Anesthesia. Anesthesia \& Analgesia 1993; 76(5): $1023 ? ? ? 6$.

21. Eroglu F, Yavuz L, Ceylan BG, Sevin G, Soyupek S. Prophylactic Effects of Systemic Oral Ephedrine in Spinal Anesthesia-induced Hypotension during Transurethral Prostatectomy. Scandinavian Journal of Urology and Nephrology 2003; 37(2): 145-50. 